Pacific

Journal of

Mathematics

\title{
NONORIENTABLE LAGRANGIAN COBORDISMS BETWEEN LEGENDRIAN KNOTS
}

\author{
Orsola CAPOVILLA-SEARLE AND LiSA TRAYNOR
}




\title{
NONORIENTABLE LAGRANGIAN COBORDISMS BETWEEN LEGENDRIAN KNOTS
}

\author{
Orsola CAPOVILla-SEARLE AND LISA TRAYNOR
}

\begin{abstract}
In the symplectization of standard contact 3 -space, $\mathbb{R} \times \mathbb{R}^{3}$, it is known that an orientable Lagrangian cobordism between a Legendrian knot and itself, also known as an orientable Lagrangian endocobordism for the Legendrian knot, must have genus 0 . We show that any Legendrian knot has a nonorientable Lagrangian endocobordism, and that the cross-cap genus of such a nonorientable Lagrangian endocobordism must be a positive multiple of 4 . The more restrictive exact, nonorientable Lagrangian endocobordisms do not exist for any exactly fillable Legendrian knot but do exist for any stabilized Legendrian knot. Moreover, the relation defined by exact, nonorientable Lagrangian cobordism on the set of stabilized Legendrian knots is symmetric and defines an equivalence relation, a contrast to the nonsymmetric relation defined by orientable Lagrangian cobordisms.
\end{abstract}

\section{Introduction}

Smooth cobordisms are a common object of study in topology. Motivated by ideas in symplectic field theory [Eliashberg et al. 2000], Lagrangian cobordisms that are cylindrical over Legendrian submanifolds outside a compact set have been an active area of research interest. Throughout this paper, we will study Lagrangian cobordisms in the symplectization of the standard contact $\mathbb{R}^{3}$, namely the symplectic manifold $\left(\mathbb{R} \times \mathbb{R}^{3}, d\left(e^{t} \alpha\right)\right)$, where $\alpha=d z-y d x$, that coincide with the cylinders $\mathbb{R} \times \Lambda_{+}$(resp., $\mathbb{R} \times \Lambda_{-}$) when the $\mathbb{R}$-coordinate is sufficiently positive (resp., negative). Our focus will be on nonorientable Lagrangian cobordisms between Legendrian knots $\Lambda_{+}$and $\Lambda_{-}$and nonorientable Lagrangian endocobordisms, which are nonorientable Lagrangian cobordisms with $\Lambda_{+}=\Lambda_{-}$.

Smooth endocobordisms in $\mathbb{R} \times \mathbb{R}^{3}$ without the Lagrangian condition are abundant: for any smooth knot $K \subset \mathbb{R}^{3}$ and an arbitrary $j \geq 0$, there is a smooth 2-dimensional orientable submanifold $M$ of genus $j$ such that $M$ agrees with the cylinder $\mathbb{R} \times K$ when the $\mathbb{R}$-coordinate lies outside an interval $\left[T_{-}, T_{+}\right]$; the analogous statement

MSC2010: primary 57R17, 53D42; secondary 57M25.

Keywords: Legendrian knot, Lagrangian cobordism, Lagrangian endocobordism, exact Lagrangian, fillable Legendrian. 
holds for nonorientable $M$ and cross-cap genus ${ }^{1}$ when $j>0$. For any Legendrian knot $\Lambda$, it is easy to construct an orientable Lagrangian endocobordism of genus 0 , namely the trivial Lagrangian cylinder $\mathbb{R} \times \Lambda$. In fact, with the added Lagrangian condition, orientable Lagrangian endocobordisms must be concordances:

Theorem [Chantraine 2010]. Any orientable Lagrangian endocobordism of any Legendrian knot has genus 0.

Nonorientable Lagrangian endocobordisms also exist and have topological restrictions:

Theorem 1.1. For an arbitrary Legendrian knot $\Lambda$, there exists a nonorientable Lagrangian endocobordism for $\Lambda$ of cross-cap genus $g$ if and only if $g \in 4 \mathbb{Z}^{+}$.

Theorem 1.1 is proved in Theorems 3.2 and 3.3. The fact that the cross-cap genus of a nonorientable Lagrangian endocobordism must be a positive multiple of 4 follows from a result of Audin [1988] about the obstruction to the Euler characteristic for closed, Lagrangian submanifolds in $\mathbb{R}^{4}$. It is easy to construct immersed Lagrangian endocobordisms; the existence of the desired embedded endocobordisms follows from Lagrangian surgery, as developed, for example, by Polterovich [1991].

Of special interest are Lagrangian cobordisms that satisfy an additional "exactness" condition. Exactness is known to be quite restrictive: by a foundational result of Gromov [1985], there are no closed, exact Lagrangian submanifolds in $\mathbb{R}^{2 n}$ with its standard symplectic structure. The nonclosed trivial Lagrangian cylinder $\mathbb{R} \times \Lambda$ is exact, and Section 2 describes some general methods to construct exact Lagrangian cobordisms. In contrast to Theorem 1.1, there are some Legendrians that do not admit exact, nonorientable Lagrangian endocobordisms:

Theorem 1.2. There does not exist an exact, nonorientable Lagrangian endocobordism for any Legendrian knot $\Lambda$ that is exactly orientably or nonorientably fillable.

A Legendrian knot $\Lambda$ is exactly fillable if there exists an exact Lagrangian cobordism that is cylindrical over $\Lambda$ at the positive end and does not intersect $\left\{T_{-}\right\} \times \mathbb{R}^{3}$ for $T_{-} \ll 0$; precise definitions can be found in Section 2. Theorem 1.2 is proved in Section 4; it follows from the Seidel isomorphism (Theorem 4.1), which relates the topology of a filling to the linearized contact cohomology of the Legendrian at the positive end. Theorem 1.2 implies that on the set of Legendrian knots in $\mathbb{R}^{3}$ that are exactly fillable, orientably or not, the relation defined by exact, nonorientable Lagrangian cobordism is antireflexive and antisymmetric; see Corollary 4.2. Figure 6 gives some particular examples of Legendrians that are exactly fillable and thus do not admit exact, nonorientable Lagrangian endocobordisms. Many of these examples are maximal tb Legendrian representatives of twist or torus knots. In fact,

\footnotetext{
${ }^{1}$ the number of real projective planes in a connected sum decomposition
} 


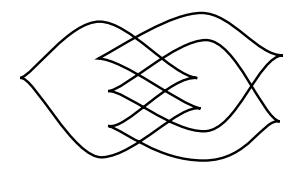

Figure 1. The max tb Legendrian representative of $m\left(8_{19}\right)$.

using the classification results of Etnyre and Honda [2001] and of Etnyre, $\mathrm{Ng}$, and Vértesi [Etnyre et al. 2013], we show:

Corollary 1.3. Let $K$ be the smooth knot type of either a twist knot or a positive torus knot or a negative torus knot of the form $T(-p, 2 k)$ for $p$ odd and $p>2 k>0$. Then any maximal tb Legendrian representative of $K$ does not have an exact, nonorientable Lagrangian endocobordism.

However, stabilized Legendrian knots do admit exact, nonorientable Lagrangian endocobordisms: a Legendrian knot is said to be stabilized if, after Legendrian isotopy, a strand contains a zig-zag as shown in Figure 4.

Theorem 1.4. For any stabilized Legendrian knot $\Lambda$ and any $k \in \mathbb{Z}^{+}$, there exists an exact, nonorientable Lagrangian endocobordism for $\Lambda$ of cross-cap genus $4 k$.

Some Legendrian knots are neither exactly fillable nor stabilized. Thus, a natural question is:

Question 1.5. If a Legendrian knot is not exactly fillable and is not stabilized, does it have an exact, nonorientable Lagrangian endocobordism? In particular, does the unique Legendrian representative of $m\left(8_{19}\right)=T(-4,3)$ with maximal tb whose front projection is shown in Figure 1 have an exact, nonorientable Lagrangian endocobordism?

A description of how the Legendrian knot can be recovered from the front projection is given on page 322 . The max tb version of $m\left(8_{19}\right)$ is not exactly fillable since the upper bound on the tb invariant for all Legendrian representatives of $m\left(8_{19}\right)$ given by the Kauffman polynomial is not sharp; see Section 6 for more details. In response to Question 1.5, Chantraine, Dimitroglou Rizell, Ghiggini, and Golovko [Chantraine et al. 2015, Corollary 12.3] proved an extension of Theorem 1.2 that shows an exact, nonorientable Lagrangian endocobordism does not exist for an orientable Legendrian that admits an augmentation or, more generally, for an orientable Legendrian whose characteristic algebra admits a finite-dimensional representation. The max tb Legendrian representative of $m\left(8_{19}\right)=T(-4,3)$ does not have an augmentation, but by results of Sivek [2013, Corollary 3.5], the characteristic algebra of this Legendrian does have a 2-dimensional representation. Thus the answer to Question 1.5 is no; see Section 6 for additional questions.

Given the existence of exact, nonorientable Lagrangian endocobordisms for a stabilized Legendrian, it is natural to ask: What Legendrian knots can appear as a 
"slice" of such an endocobordism? The parallel question for orientable Lagrangian endocobordisms has been studied in [Chantraine 2015; Baldwin and Sivek 2014; Cornwell et al. 2016]. The nonorientable version of this question is closely tied to the question of whether or not nonorientable Lagrangian cobordisms define an equivalence relation on the set of Legendrian knots. By a result of Chantraine [2010], it is known that the relation defined on the set of Legendrian knots by orientable Lagrangian cobordism is not an equivalence relation since symmetry fails. In fact, the relation defined on the set of stabilized Legendrian knots by exact, nonorientable Lagrangian cobordism is symmetric: see Theorem 5.2. In addition, this relation is transitive by "stacking" (Lemma 2.2) and reflexive by Theorem 1.4. Thus we get:

Theorem 1.6. On the set of stabilized Legendrian knots, the relation defined by exact, nonorientable Lagrangian cobordism is an equivalence relation. Moreover, all stabilized Legendrian knots are equivalent with respect to this relation.

\section{Background}

In this section we review Legendrian and Lagrangian submanifolds.

Contact manifolds and Legendrian submanifolds. Below is some basic background on contact manifolds and Legendrian knots. More information can be found, for example, in [Etnyre 2003; 2005].

A contact manifold $(Y, \xi)$ is an odd-dimensional manifold together with a contact structure, which consists of a maximally nonintegrable field of tangent hyperplanes. The standard contact structure on $\mathbb{R}^{3}$ is the field $\xi_{p}=\operatorname{ker} \alpha_{0}(p)$ for $\alpha_{0}(x, y, z)=$ $d z-y d x$. A Legendrian link $\Lambda$ is a submanifold of $\mathbb{R}^{3}$ diffeomorphic to a disjoint union of circles such that for all $p \in \Lambda$, we have $T_{p} \Lambda \subset \xi_{p}$; if, in addition, $\Lambda$ is connected, $\Lambda$ is a Legendrian knot. It is common to examine Legendrian links from their $x z$-projections, known as their front projections. A Legendrian link will generically have an immersed front projection with semicubical cusps, no vertical tangents, and no self-tangencies; any such projection can be uniquely lifted to a Legendrian link using $y=d z / d x$.

Two Legendrian links $\Lambda_{0}$ and $\Lambda_{1}$ are equivalent Legendrian links if there exists a 1-parameter family of Legendrian links $\Lambda_{t}, t \in[0,1]$, joining $\Lambda_{0}$ and $\Lambda_{1}$. In fact, Legendrian links $\Lambda_{0}, \Lambda_{1}$ are equivalent if and only if their front projections are equivalent by planar isotopies that do not introduce vertical tangents and the Legendrian Reidemeister moves as shown in Figure 2.

Every knot has a Legendrian representative. In fact, every knot has an infinite number of different Legendrian representatives. For example, Figure 3 shows three different oriented Legendrians that are all topologically the unknot. These unknots can be distinguished by classical Legendrian invariants: the Thurston-Bennequin 


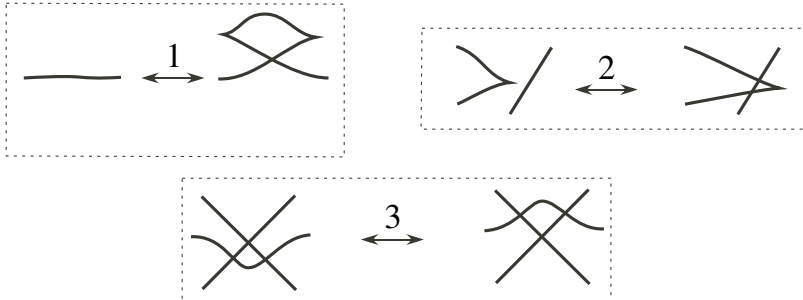

Figure 2. The three Legendrian Reidemeister moves. There is another type-1 move obtained by flipping the planar figure about a horizontal line, and there are three additional type- 2 moves obtained by flipping the planar figure about a vertical, a horizontal, and both a vertical and a horizontal line.
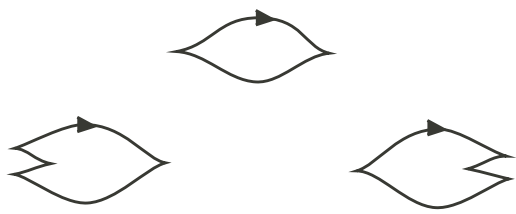

Figure 3. Three different Legendrian unknots: the one with maximal tb invariant of -1 and two others obtained by \pm -stabilizations.

number, tb, and the rotation number, $r$. These invariants can easily be computed from a front projection; see, for example, [Boranda et al. 2013].

The two unknots in the second line of Figure 3 are obtained from the one at the top by stabilization. In general, from an oriented Legendrian $\Lambda$, one can obtain oriented Legendrians $S_{ \pm}(\Lambda)$ : the positive (negative) stabilization, $S_{+}\left(S_{-}\right)$, is obtained by replacing a portion of a strand with a strand that contains a down (up) zig-zag, as shown in Figure 4. This stabilization procedure will not change the underlying smooth knot type but will decrease the Thurston-Bennequin number by 1 ; adding an up (down) zig-zag will decrease (increase) the rotation number by 1 . It is possible to move a zig-zag to any strand of a Legendrian knot [Fuchs and Tabachnikov 1997]. Bennequin and slice-Bennequin inequalities (see, for example, [Etnyre 2005]) show that for any Legendrian representative $\Lambda$ of a fixed smooth knot type $K, \operatorname{tb}(\Lambda)+|r(\Lambda)|$ is bounded above. Because of such bounds, the set of oriented Legendrian representatives of a fixed smooth knot type can be visualized by a "mountain range" in the plane where each Legendrian representative $\Lambda$ is recorded by a vertex at coordinates $(r(\Lambda), \mathrm{tb}(\Lambda))$; two vertices are connected by an edge if the corresponding Legendrians are related by stabilization. Many examples of known and conjectured mountain ranges can be found in the Legendrian knot atlas of Chongchitmate and $\mathrm{Ng}$ [2013]. 


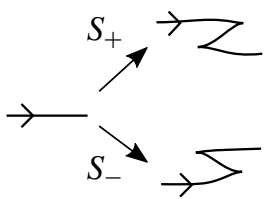

Figure 4. The positive (negative) stabilization of an oriented knot is obtained by introducing a down (an up) zig-zag.

\section{Symplectic manifolds, Lagrangian submanifolds, and Lagrangian cobordisms.}

We will now discuss some basic concepts in symplectic geometry. Additional background can be found, for example, in [McDuff and Salamon 1998].

A symplectic manifold $(M, \omega)$ is an even-dimensional manifold together with a 2 -form $\omega$ that is closed and nondegenerate; when $\omega$ is an exact 2-form, $(M, \omega=d \beta)$ is said to be an exact symplectic manifold. A basic example of an exact symplectic manifold is $\left(\mathbb{R}^{4}, \omega_{0}=d x_{1} \wedge d y_{1}+d x_{2} \wedge d y_{2}\right)$. The cobordisms constructed in this paper live inside the symplectic manifold that is constructed as the symplectization of $\left(\mathbb{R}^{3}, \xi_{0}=\operatorname{ker} \alpha_{0}\right)$, namely, $\mathbb{R} \times \mathbb{R}^{3}$ with symplectic form given by $\omega=d\left(e^{t} \alpha_{0}\right)$. In fact, there is an exact symplectic diffeomorphism between the symplectization $\left(\mathbb{R} \times \mathbb{R}^{3}, \omega\right)$ and the standard $\left(\mathbb{R}^{4}, \omega_{0}\right)$; see, for example, [Bourgeois et al. 2015].

A Lagrangian submanifold $L$ of a 4-dimensional symplectic manifold $(M, \omega)$ is a 2-dimensional submanifold such that $\left.\omega\right|_{L}=0$. When $M$ is an exact symplectic manifold, $\omega=d \beta,\left.\beta\right|_{L}$ is necessarily a closed 1-form; when, in addition, $\left.\beta\right|_{L}$ is an exact 1 -form, $\left.\beta\right|_{L}=d f$, then $L$ is said to be an exact Lagrangian submanifold. It is easy to verify that the exactness of the Lagrangian does not depend on the choice of $\beta$.

Remark. There is a (nonexact) Lagrangian torus in the standard symplectic $\mathbb{R}^{4}$ : this can be seen as the product of two embedded circles in each of the $\left(x_{1}, y_{1}\right)$ and $\left(x_{2}, y_{2}\right)$ planes. By classical algebraic topology, it follows that the torus is the only compact, orientable surface that admits a Lagrangian embedding into $\mathbb{R}^{4}$ : a result of Whitney equates a signed count of double points of an immersion to the Euler number of the normal bundle, but for a Lagrangian submanifold, the normal and tangent bundles are isomorphic [Audin et al. 1994].

We now turn our focus to noncompact Lagrangians that are cylindrical over Legendrians.

Definition 2.1. Let $\Lambda_{-}, \Lambda_{+}$be Legendrian links in $\mathbb{R}^{3}$.

(1) A Lagrangian submanifold without boundary $L \subset \mathbb{R} \times \mathbb{R}^{3}$ is a Lagrangian cobordism from $\Lambda_{+}$to $\Lambda_{-}$if it is of the form

$$
L=\left(\left(-\infty, T_{-}\right] \times \Lambda_{-}\right) \cup \bar{L} \cup\left(\left[T_{+},+\infty\right) \times \Lambda_{+}\right)
$$


for some $T_{-}<T_{+}$, where $\bar{L} \subset\left[T_{-}, T_{+}\right] \times \mathbb{R}^{3}$ is compact with boundary $\partial \bar{L}=\left(\left\{T_{-}\right\} \times \Lambda_{-}\right) \cup\left(\left\{T_{+}\right\} \times \Lambda_{+}\right)$.

(2) A Lagrangian cobordism from $\Lambda_{+}$to $\Lambda_{-}$is orientable (resp., nonorientable) if $L$ is orientable (resp., nonorientable).

(3) A Lagrangian cobordism from $\Lambda_{+}$to $\Lambda_{-}$is exact if $L$ is exact, namely $\left.e^{t} \alpha_{0}\right|_{L}=$ $\left.d f\right|_{L}$, and the primitive, $f$, is constant on the cylindrical ends: there exist constants $C_{ \pm}$such that

$$
\left.f\right|_{L \cap\left(\left(-\infty, T_{-}\right) \times \mathbb{R}^{3}\right)}=C_{-},\left.\quad f\right|_{L \cap\left(\left(T_{+},+\infty\right) \times \mathbb{R}^{3}\right)}=C_{+} .
$$

A Legendrian $\operatorname{knot} \Lambda$ is (exactly) fillable if there exists an (exact) Lagrangian cobordism from $\Lambda_{+}=\Lambda$ to $\Lambda_{-}=\varnothing$.

An important property of Lagrangian cobordisms is that they can be stacked (or composed):

Lemma 2.2 (stacking cobordisms [Ekholm et al. 2012]). If $L_{12}$ is a Lagrangian cobordism from $\Lambda_{+}=\Lambda_{1}$ to $\Lambda_{-}=\Lambda_{2}$, and $L_{23}$ is a Lagrangian cobordism from $\Lambda_{+}=\Lambda_{2}$ to $\Lambda_{-}=\Lambda_{3}$, then there exists a Lagrangian cobordism $L_{13}$ from $\Lambda_{+}=\Lambda_{1}$ to $\Lambda_{-}=\Lambda_{3}$. Furthermore, if $L_{12}$ and $L_{23}$ are exact, then there exists an exact $L_{13}$.

Constructions of exact Lagrangian cobordisms are an active area of research. In this paper, we will use the fact that there exist exact Lagrangian cobordisms between Legendrians related by isotopy and certain surgeries. The existence of exact Lagrangian cobordisms from isotopy is well known; see, for example, [Eliashberg and Gromov 1998; Chantraine 2010; Ekholm et al. 2012; Bourgeois et al. 2015].

Lemma 2.3 (exact cobordisms from isotopy). Suppose that $\Lambda$ and $\Lambda^{\prime}$ are isotopic Legendrian links. Then there exists an exact, orientable Lagrangian cobordism, in fact concordance, from $\Lambda_{+}=\Lambda$ to $\Lambda_{-}=\Lambda^{\prime}$.

Remark. In general, the trace of a Legendrian isotopy is not a Lagrangian cobordism. However it is possible to add a "correction term" so that it will be Lagrangian. More precisely, let $\lambda_{t}(u)=(x(t, u), y(t, u), z(t, u)), t \in \mathbb{R}$, be a Legendrian isotopy such that $\frac{\partial \lambda}{\partial t}(t, u)$ has compact support with $\operatorname{Im} \lambda_{t}(u)=\Lambda_{-}$for $t \leq-T$ and $\operatorname{Im} \lambda_{t}(u)=\Lambda_{+}$for $t \geq T$, and let

$$
\eta(t, u)=\alpha_{0}\left(\frac{\partial \lambda}{\partial t}(t, u)\right) .
$$

Then $\Gamma(t, u)=(t, x(t, u), y(t, u), z(t, u)+\eta(t, u))$ is an exact Lagrangian immersion. If $\eta(t, u)$ is sufficiently small, which can be guaranteed by "slowing down" the isotopy via a $t$-reparametrization, then $\Gamma(t, u)$ is an exact Lagrangian embedding.

In addition, Legendrians $\Lambda$ and $\Lambda^{\prime}$ that differ by "surgery" can be connected by an exact Lagrangian cobordism. In one of these surgery operations, a Legendrian 


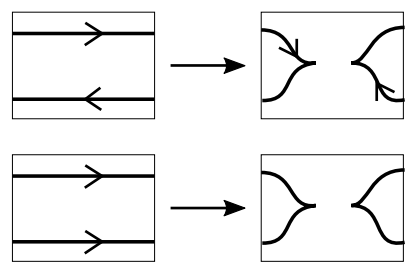

Figure 5. Orientable and nonorientable Legendrian surgeries.

0-tangle, consisting of two strands with no crossings and no cusp points, is replaced with a Legendrian $\infty$-tangle, consisting of two strands that each have one cusp and no crossings; see Figure 5. When the strands of the 0 -tangle are oppositely oriented, this is an orientable surgery; otherwise this is a nonorientable surgery. There is another surgery operation that shows that the maximal tb Legendrian representative of the unknot, shown at the top of Figure 3, can be filled.

Lemma 2.4 (exact cobordisms from surgery [Ekholm et al. 2012; Dimitroglou Rizell 2014; Bourgeois et al. 2015]).

(1) Suppose $\Lambda_{+}$and $\Lambda_{-}$are Legendrian knots, where $\Lambda_{-}$is obtained from $\Lambda_{+}$by orientable (nonorientable) surgery, as shown in Figure 5. Then there exists an exact, orientable (nonorientable) Lagrangian cobordism from $\Lambda_{+}$to $\Lambda_{-}$.

(2) Suppose $\Lambda_{+}$is the Legendrian unknot with tb equal to the maximum value of -1 . Then there exists an exact, orientable Lagrangian filling of $\Lambda_{+}$by a disk.

Remark. By Lemmas 2.2, 2.3, and 2.4, to show there exists an exact Lagrangian cobordism from $\Lambda_{+}$to $\Lambda_{-}$, it suffices to show that there is a string of Legendrian links $\left(\Lambda_{+}=\Lambda_{0}, \Lambda_{1}, \ldots, \Lambda_{n}=\Lambda_{-}\right)$, where each $\Lambda_{i+1}$ is obtained from $\Lambda_{i}$ by a single surgery, as shown in Figure 5, and Legendrian isotopy. In the case where each surgery is orientable, the exact Lagrangian cobordism will be orientable. If all surgeries are orientable and $\Lambda_{ \pm}$are both knots, then the length, $n$, of this string must be even and will agree with twice the genus of the Lagrangian cobordism; for more details, see [Boranda et al. 2013]. If there is at least one nonorientable surgery, the exact Lagrangian cobordism will be nonorientable and the length of the string agrees with the cross-cap genus of the Lagrangian cobordism. To construct an exact Lagrangian filling of $\Lambda_{+}$, it suffices to construct such a string to $\Lambda_{-}=U$, where $U$ is a trivial link of maximal tb Legendrian unknots.

\section{Constructions of nonorientable Lagrangian endocobordisms}

We show that any Legendrian knot has a nonorientable Lagrangian endocobordism with cross-cap genus an arbitrary multiple of 4 . We then show that it is not possible to get any other cross-cap genera. 
The strategy to show existence is to first construct an immersed orientable Lagrangian cobordism, and then apply "Lagrangian surgery" to modify it so that it is embedded. The following description of Lagrangian surgery follows Polterovich's construction [1991]; see also [Lalonde and Sikorav 1991].

To describe Lagrangian surgery precisely, we first need to explain the "sign" of a double point. Suppose that $x$ is a point of self-intersection of a generic, immersed, oriented 2-dimensional submanifold $L$ of $\mathbb{R}^{4}$. Then $\operatorname{sgn}(x) \in\{ \pm 1\}$ will denote the sign of self-intersection of $L$ at $x$ : let $\left(v_{1}, v_{2}\right)$ and $\left(w_{1}, w_{2}\right)$ be positively oriented bases of the transverse tangent spaces at $x$; then

$$
\operatorname{sgn}(x)=+1 \Longleftrightarrow\left(v_{1}, v_{2}, w_{1}, w_{2}\right) \text { is a positively oriented basis of } \mathbb{R}^{4},
$$

and otherwise $\operatorname{sgn}(x)=-1$.

By constructing a Lagrangian handle in a Darboux chart, it is possible to remove double points of a Lagrangian immersion:

Lemma 3.1 (Lagrangian surgery [Polterovich 1991]). Let $\Sigma$ be a 2-dimensional manifold. Suppose $\phi: \Sigma \rightarrow \mathbb{R}^{4}$ is a Lagrangian immersion and $U \subset \mathbb{R}^{4}$ contains a single transversal double point $x$ of $\phi$. Then there exists a 2-dimensional manifold $\Sigma^{\prime}$ and a Lagrangian immersion $\phi^{\prime}: \Sigma^{\prime} \rightarrow \mathbb{R}^{4}$ such that

(1) $\operatorname{Im} \phi=\operatorname{Im} \phi^{\prime}$ on $\mathbb{R}^{4}-U$,

(2) $\phi^{\prime}$ has no double points in $U$.

Furthermore, let $\phi^{-1}(\{x\})=\left\{p_{1}, p_{2}\right\} \subset \Sigma$.

(1) If $p_{1}, p_{2}$ are in disjoint components of $\Sigma$, then $\Sigma^{\prime}$ is obtained from $\Sigma$ by a connect sum operation.

(2) If $p_{1}, p_{2}$ are in the same component of $\Sigma$, then

(a) if $\Sigma$ is not orientable, $\Sigma^{\prime}=\Sigma \# K(=\Sigma \# T)$,

(b) if $\Sigma$ is orientable, $\Sigma^{\prime}=\Sigma \# T$ when $\operatorname{sgn}(x)=+1$, and $\Sigma^{\prime}=\Sigma \# K$ when $\operatorname{sgn}(x)=-1$,

where $K$ denotes the Klein bottle and $T$ denotes the torus.

We now have the necessary background to show the existence of a nonorientable Lagrangian endocobordism for any Legendrian knot:

Theorem 3.2. For any Legendrian knot $\Lambda$ and any $k \in \mathbb{Z}^{+}$, there exists a nonorientable Lagrangian endocobordism for $\Lambda$ of cross-cap genus $4 k$.

Proof. For an arbitrary Legendrian knot $\Lambda$, begin with a cylindrical Lagrangian cobordism, $L=\mathbb{R} \times \Lambda$ in $\mathbb{R} \times \mathbb{R}^{3}$, which is a space that is symplectically equivalent to the standard $\mathbb{R}^{4}$. As explained in the remark on page 324 , there exists an embedded Lagrangian torus, $T$, such that $T \cap L=\varnothing$. After a suitable shift and perturbation, we can assume that $L$ and $T$ intersect at exactly two points, $x_{1}$ and $x_{2}$, where 
$\operatorname{sgn}\left(x_{1}\right)=+1$ and $\operatorname{sgn}\left(x_{2}\right)=-1$. By Lemma 2.4, Lagrangian surgery at $x_{1}$ results in the connected, oriented, immersed Lagrangian diffeomorphic to $\left(\mathbb{R} \times S^{1}\right) \# T$ with a double point at $x_{2}$ of index -1 ; a second Lagrangian surgery at $x_{2}$ results in an embedded, nonorientable Lagrangian cobordism diffeomorphic to $\left(\mathbb{R} \times S^{1}\right) \# T \# K$, and thus of cross-cap genus 4. Stacking these endocobordisms, using Lemma 2.2, produces an embedded, nonorientable Lagrangian cobordism of cross-cap genus $4 k$ for any $k \in \mathbb{Z}^{+}$.

In fact, the possible cross-cap genera that appear in Theorem 3.2 are all that can exist:

Theorem 3.3. Any nonorientable Lagrangian endocobordism in $\mathbb{R} \times \mathbb{R}^{3}$ must have cross-cap genus $4 k$ for some $k \in \mathbb{Z}^{+}$.

This cross-cap genus restriction is closely tied to Euler characteristic obstructions for compact, nonorientable submanifolds that admit Lagrangian embeddings in $\left(\mathbb{R}^{4}, \omega_{0}\right)$, or equivalently in $\left(\mathbb{R} \times \mathbb{R}^{3}, d\left(e^{t} \alpha\right)\right)$ :

Lemma 3.4 [Audin 1988]. Any compact, nonorientable Lagrangian submanifold of $\mathbb{R} \times \mathbb{R}^{3}$ has an Euler characteristic divisible by 4 .

This result can be seen as an extension, to the nonorientable setting, of a formula of Whitney that relates the number of double points of a smooth immersion to the Euler number of the normal bundle of the immersion; see [Audin 1988; Audin et al. 1994].

Remark. Lemma 3.4 implies that any compact, nonorientable Lagrangian submanifold $L$ in $\mathbb{R} \times \mathbb{R}^{3}$ has cross-cap genus $2+4 j$ for some $j \geq 0$. There are explicit constructions of compact, nonorientable Lagrangian submanifolds of crosscap genus $2+4 j$ for all $j>0$ [Givental 1986; Audin 1990]. It has been shown that there is no embedded, Lagrangian Klein bottle $(j=0)$ [Nemirovskii 2009; Shevchishin 2009].

To utilize the cross-cap genus restrictions for compact Lagrangians, we will employ the following lemma, which shows that for any Lagrangian endocobordism, it is possible to construct a compact, nonorientable Lagrangian submanifold into which we can glue the compact portion of a Lagrangian endocobordism.

Lemma 3.5. For any Legendrian knot $\Lambda \subset \mathbb{R}^{3}$, any open set $D \subset \mathbb{R}^{3}$ containing $\Lambda$, and any $T \in \mathbb{R}^{+}$, there exists a compact, nonorientable Lagrangian submanifold $L$ in $\mathbb{R} \times \mathbb{R}^{3}$ such that

$$
L \cap([-T, T] \times D)=[-T, T] \times \Lambda .
$$

Proof. The strategy will be to construct a Lagrangian torus with double points, thought of as two finite cylinders with top and bottom circles identified, and then 
apply Lagrangian surgery to remove the double points. As a first step, we construct (nondisjoint) Lagrangian embeddings of two cylinders via Legendrian isotopies (see Lemma 2.3). Namely, start with two disjoint copies of $\Lambda: \Lambda$ in $D$ and a translated version $\Lambda^{\prime} \subset \mathbb{R}^{3}-D$. Now, for $t \in[0, U]$, consider Legendrian isotopies $\Lambda_{t}$ of $\Lambda$ and $\Lambda_{t}^{\prime}$ of $\Lambda^{\prime}$ that satisfy the following conditions: $\Lambda_{t}=\Lambda$ for $t \in[0, U] ; \Lambda_{t}^{\prime}=\Lambda^{\prime}$ for $t \in[0, T]$; and for $t \in[T, U], \Lambda_{t}^{\prime}$ is a Legendrian isotopy of $\Lambda^{\prime}$ such that $\Lambda_{t}^{\prime}=\Lambda$ for $t$ near $U$. By repeating an analogous procedure for $t \in[-U, 0]$, we can obtain a smooth immersion of the torus into $[-U, U] \times \mathbb{R}^{3}$. The arguments used to prove Lemma 2.3 (see the remark on page 325) show that for $U-T$ sufficiently large, the trace of these isotopies can be perturbed to two nondisjoint embedded Lagrangian cylinders that do not have any intersection points in $[-T, T] \times \mathbb{R}^{3}$, and a direct calculation shows that each double point with $t \in[T, U]$ can be paired up with a double point with $t \in[-U,-T]$ of opposite sign. Then by applying Lagrangian surgery (see Lemma 3.1) at each double point we get a compact, nonorientable Lagrangian submanifold $L$ in $\mathbb{R} \times \mathbb{R}^{3}$ with the desired properties.

We are now ready to prove the cross-cap genus restriction for arbitrary nonorientable Lagrangian endocobordisms:

Proof of Theorem 3.3. Let $C$ be a nonorientable Lagrangian endocobordism. Suppose $C \subset \mathbb{R} \times D$ and $C$ agrees with a standard cylinder outside $[-T, T] \times \mathbb{R}^{3}$. By Lemma 3.5, there is a compact, nonorientable Lagrangian submanifold $L$ in $\mathbb{R} \times \mathbb{R}^{3}$ such that

$$
L \cap([-T, T] \times D)=[-T, T] \times \Lambda .
$$

Let $L^{\prime}$ be the Lagrangian submanifold obtained by removing the standard cylindrical portion of $L$ in $[-T, T] \times D$ and replacing it with $C \cap\left([-T, T] \times \mathbb{R}^{3}\right)$. Then $L^{\prime}$ will be a compact, nonorientable Lagrangian submanifold whose cross-cap genus, $k\left(L^{\prime}\right)$, differs from the cross-cap genus of $L, k(L)$, by the cross-cap genus of $C, k(C)$ : $k\left(L^{\prime}\right)=k(L)+k(C)$. By Lemma 3.4, there exist $j, j^{\prime} \in \mathbb{Z}^{+}$such that $k(L)=2+4 j$ and $k\left(L^{\prime}\right)=2+4 j^{\prime}$. Thus we find that $k(C)$ must be divisible by 4 .

Remark. For exact Lagrangian cobordisms that are constructed from isotopy and surgery (see Lemmas 2.3 and 2.4) it is possible to show that the cross-cap genus must be a multiple of 4 by an alternate argument that relies on a careful analysis of the possible changes to $\operatorname{tb}(\Lambda)$ under surgery [Capovilla-Searle 2015].

\section{Obstructions to exact, nonorientable Lagrangian endocobordisms}

We will now focus on exact, nonorientable Lagrangian cobordisms. We will prove Theorem 1.2, which states that any Legendrian knot that is exactly fillable does not have an exact, nonorientable Lagrangian endocobordism. The proof of this theorem will involve applying the Seidel isomorphism, which relates the topology of a filling 
to the linearized Legendrian contact cohomology of the Legendrian at the positive end. We will then apply Theorem 1.2 and give examples of maximal tb Legendrian knots that do not have exact, nonorientable Lagrangian endocobordisms.

We begin with a brief description of Legendrian contact homology; additional background information can be found, for example, in [Etnyre 2005]. Legendrian contact homology is a Floer-type invariant of a Legendrian submanifold that lies within Eliashberg, Givental, and Hofer's symplectic field theory framework [Eliashberg 1998; Eliashberg et al. 2000; Chekanov 2002]. It is possible to associate to a Legendrian submanifold $\Lambda \subset \mathbb{R}^{3}$ the stable, tame isomorphism class of an associative differential graded algebra (DGA), $(\mathcal{A}(\Lambda), \partial)$. The algebra is freely generated by the so-called Reeb chords of $\Lambda$, and is graded using a Maslov index. The differential comes from counting pseudoholomorphic curves in the symplectization of $\mathbb{R}^{3}$; for our interests, we will always use $\mathbb{Z} / 2$ coefficients. Legendrian contact homology, namely the homology of $(\mathcal{A}(\Lambda), \partial)$, is a Legendrian isotopy invariant. Legendrian contact homology has been defined for Legendrians in contact manifolds other than $\mathbb{R}^{3}$; see, for example, [Ekholm et al. 2007; Sabloff 2003].

In general, it is difficult to extract information directly from the Legendrian contact homology. An important computational technique arises from the existence of augmentations of the DGA. An augmentation $\varepsilon$ of $\mathcal{A}(\Lambda)$ is a differential algebra homomorphism $\varepsilon:(\mathcal{A}(\Lambda), \partial) \rightarrow\left(\mathbb{Z}_{2}, 0\right)$; a graded augmentation is an augmentation such that $\varepsilon$ is supported on elements of degree 0 . Not all Legendrians have an augmentation; for any Legendrian $\Lambda$, there are only a finite number of augmentations. Given a graded augmentation $\varepsilon$, one can linearize $(\mathcal{A}(\Lambda), \partial)$ to a finitedimensional differential graded complex $\left(A(\Lambda), \partial^{\varepsilon}\right)$ and obtain linearized contact homology, denoted $\operatorname{LCH}_{*}(\Lambda, \varepsilon ; \mathbb{Z} / 2)$, and its dual linearized contact cohomology, $\mathrm{LCH}^{*}(\Lambda, \varepsilon ; \mathbb{Z} / 2)$. The set of all linearized (co)homology groups with respect to all possible graded augmentations is an invariant of $\Lambda$. If the augmentation is ungraded, one can still examine the rank of the nongraded linearized (co)homology, $\operatorname{dim} \operatorname{LCH}(\Lambda, \varepsilon ; \mathbb{Z} / 2)$, and obtain as an invariant of $\Lambda$ the set of ranks of this total linearized (co)homology for all possible augmentations. Ungraded linearized (co)homology is not an effective invariant: of the many examples of Legendrian knots in the Legendrian knot atlas of Chongchitmate and $\mathrm{Ng}$ [2013] that have the same classical invariants yet can be distinguished through graded linearized homology, none can be distinguished by examining ungraded homology. However, ungraded (co)homology will be useful in arguments below.

Ekholm [2008] has shown that an exact Lagrangian filling, $F$, of a Legendrian submanifold $\Lambda \subset \mathbb{R}^{3}$ induces an augmentation $\varepsilon_{F}$ of $(\mathcal{A}(\Lambda), \partial)$. When this filling has Maslov class 0 , the augmentation will be graded. Informally, Maslov 0 means that along each loop in the filling, the corresponding loop of Lagrangian tangent planes is trivial in the Lagrangian Grassmannian. 
The following result of Seidel will play a central role in showing obstructions to exact, nonorientable Lagrangian endocobordisms. A proof of this result was sketched in [Ekholm 2012] and given in detail in [Dimitroglou Rizell 2016]; a parallel result using generating family homology was given in [Sabloff and Traynor 2013].

Theorem 4.1 (Seidel isomorphism [Ekholm 2012; Dimitroglou Rizell 2016; Ekholm et al. 2012]). Let $\Lambda \subset \mathbb{R}^{2 n+1}$ be an $n$-dimensional Legendrian submanifold with an exact Lagrangian filling $F$; let $\varepsilon_{F}$ denote the augmentation induced by the filling. Then

$$
\operatorname{dim} H(F ; \mathbb{Z} / 2)=\operatorname{dim} \operatorname{LCH}\left(\Lambda, \varepsilon_{F} ; \mathbb{Z} / 2\right) .
$$

If the filling $F$ of the $n$-dimensional Legendrian has Maslov class 0 , then a graded version of the above equality holds:

$$
\operatorname{dim} H_{n-*}(F ; \mathbb{Z} / 2)=\operatorname{dim} \operatorname{LCH}^{*}\left(\Lambda, \varepsilon_{F} ; \mathbb{Z} / 2\right) .
$$

The ungraded version of the Seidel isomorphism will be used to prove that any Legendrian $\Lambda$ that is exactly fillable does not have an exact, nonorientable Lagrangian endocobordism:

Proof of Theorem 1.2. For a contradiction, suppose that there is a Legendrian knot $\Lambda$ that has an exact Lagrangian filling and an exact, nonorientable Lagrangian endocobordism. Then by stacking the endocobordisms (see Lemma 2.2) it follows that $\Lambda$ has an infinite number of topologically distinct exact, nonorientable Lagrangian fillings. Each of these exact Lagrangian fillings induces an augmentation. Since there are only a finite number of possible augmentations, there must exist two topologically distinct fillings that induce the same augmentation. However, this gives a contradiction to the Seidel isomorphism, Theorem 4.1.

Theorem 1.2 implies that on the set of Legendrian knots in $\mathbb{R}^{3}$ that are exactly fillable, orientably or not, the relation defined by exact, nonorientable Lagrangian cobordism is antireflexive. Thus, by stacking (Lemma 2.2) we immediately also see:

Corollary 4.2. On the set of Legendrian knots in $\mathbb{R}^{3}$ that are exactly fillable, orientably or not, the relation $\sim$ defined by exact, nonorientable Lagrangian cobordism is antisymmetric: $\Lambda_{1} \sim \Lambda_{2} \Rightarrow \Lambda_{2} \nsim \Lambda_{1}$.

We now apply Theorem 1.2 to give examples of Legendrians that do not have exact, nonorientable Lagrangian endocobordisms. Hayden and Sabloff [2015] showed that every positive knot type has a Legendrian representative that has an exact, orientable Lagrangian filling. Combining this with Theorem 1.2 immediately gives the next result.

Corollary 4.3 [Hayden and Sabloff 2015]. Each positive knot has a Legendrian representative that does not have an exact, nonorientable Lagrangian endocobordism. 

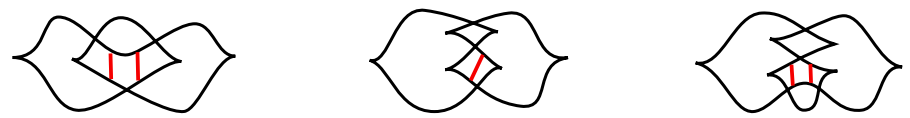

$$
m\left(3_{1}\right)=T(3,2)=K_{-2} \quad 3_{1}=T(-3,2)=K_{1} \quad 4_{1}=K_{2}=K_{-3}
$$

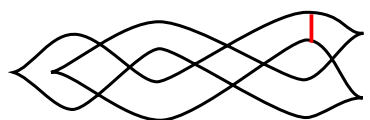

$5_{1}=T(-5,2)$

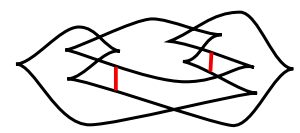

$6_{2}$

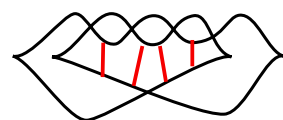

$m\left(5_{1}\right)=T(5,2)$

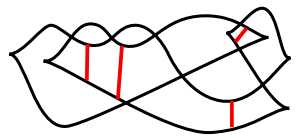

$m\left(6_{2}\right)$

Figure 6. Examples of max tb Legendrians that do not have exact, nonorientable Lagrangian endocobordisms. The red lines indicate points for surgeries.

There is work in progress to show that every +-adequate knot has a Legendrian representative with an exact filling (J. M. Sabloff, private communication).

Many maximal tb representatives of low crossing knots have fillings, orientable or not. Figure 6 illustrates some Legendrians that can be verified to have exact, Lagrangian fillings; see the remark on page 326. Many of the examples in Figure 6 are Legendrian representatives of twist knots, $K_{m}$, or torus knots, $T(p, q)$. Using Theorem 1.2 together with classification results of Etnyre and Honda [2001] and of Etnyre, $\mathrm{Ng}$, and Vértesi [Etnyre et al. 2013], we show that all maximal tb representatives of twist knots, positive torus knots, and negative torus knots of the form $T(-p, 2 k), p>2 k>0$, do not have exact, nonorientable Lagrangian endocobordisms:

Proof of Corollary 1.3. By Theorem 1.2, to show the nonexistence of an exact, nonorientable Lagrangian endocobordism, it suffices to show the existence of an exact Lagrangian filling.

First consider the case where $\Lambda$ is a maximal tb representative of a twist knot, whose form is shown in Figure 7. Etnyre, $\mathrm{Ng}$, and Vértesi [Etnyre et al. 2013] have

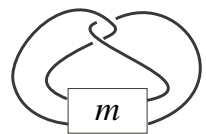

Figure 7. The smooth twist knot $K_{m}$; the box contains $m$ righthanded half-twists if $m \geq 0$, and $|m|$ left-handed twists if $m<0$. Notice that $K_{0}$ and $K_{-1}$ are unknots. 


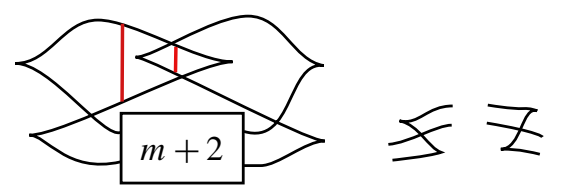

Figure 8. Any maximal tb Legendrian representative of a negative twist knot $K_{m}$, with $m \leq-2$, is Legendrian isotopic to a Legendrian of the form on the left with the box containing $|m+2|$ half-twists, where each half-twist has form $S$ (middle) or form $Z$ (right). Two surgeries produces a max tb Legendrian unknot.
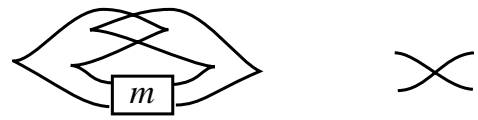

Figure 9. Any maximal tb Legendrian representative of a positive twist knot $K_{m}$, with $m \geq 1$, is Legendrian isotopic to a Legendrian in the form at left, where the box contains $m$ half-twists of form $X$, right.
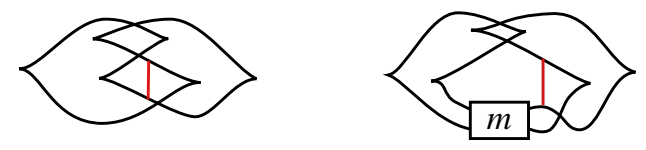

Figure 10. An inductive argument shows that every max tb representative of a positive twist knot has an exact Lagrangian filling.

classified all Legendrian twist knots: every maximal tb Legendrian representative of $K_{m}$, for $m \leq-2$, is Legendrian isotopic to one of the form in Figure 8, and every maximal tb Legendrian representative of $K_{m}$, for $m \geq 1$, is Legendrian isotopic to one of the form in Figure 9. For a max tb representative of a negative twist knot, Figure 8 illustrates the two surgeries that show the existence of an exact Lagrangian filling. For a max tb Legendrian representative of a positive twist knot, the existence of an exact filling can be shown by an induction argument: Figure 10 (left) indicates the surgery point when $m=1$; for all $m \geq 1$, a maximal tb representative of $K_{m+1}$ can be reduced to a maximal tb representative of $K_{m}$ by one surgery, as indicated in Figure 10 (right).

Next consider maximal tb Legendrian representatives of a torus knot, a knot that can be smoothly isotoped so that it lies on the surface of an unknotted torus in $\mathbb{R}^{3}$. Every torus knot can be specified by a pair $(p, q)$ of coprime integers: we will use the convention that the $(p, q)$-torus knot, $T(p, q)$, winds $p$ times around a meridional curve of the torus and $q$ times in the longitudinal direction. In fact, 


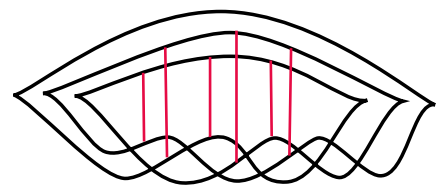

Figure 11. Surgeries that result in an exact filling of the maximal tb representative of the positive torus knot $T(5,3)$.

$T(p, q)$ is equivalent to $T(q, p)$ and to $T(-p,-q)$. We will always assume that $|p|>q \geq 2$, since we are interested in nontrivial torus knots.

Etnyre and Honda [2001] showed there is a unique maximal tb representative of a positive torus knot $T(p, q)$ with $p>0$. The surgeries used in [Boranda et al. 2013, Theorem 4.2] show that this maximal representative is exactly fillable. Figure 11 illustrates the orientable surgeries for the $(5,3)$-torus knot; in this sequence of surgeries, one begins with surgeries on the innermost strands, and then performs a Legendrian isotopy so that it is possible to do a surgery on the next set of innermost strands.

Lastly consider the case where $\Lambda$ is topologically a negative torus knot, $T(-p, 2 k)$ with $p>2 k>0$. In this case, Etnyre and Honda have shown that the number of different maximal tb Legendrian representations depends on the divisibility of $p$ by $2 k$ : if $|p|=m 2 k+e, 0<e<2 k$, there are $m$ nonoriented Legendrian representatives of $T(-p, 2 k)$ with maximal tb. These different representatives with maximal tb are obtained by writing $m=1+n_{1}+n_{2}$, where $n_{1}, n_{2} \geq 0$, and then $\Lambda_{\left(n_{1}, n_{2}\right)}$ is constructed using the form shown in Figure 12 with $n_{1}$ and $n_{2}$ copies of the tangle $B$ inserted as indicated; this figure also shows $k$ surgeries that guarantee the existence of an exact Lagrangian filling.

Some comments on obstructions to exact fillings are discussed in Section 6.

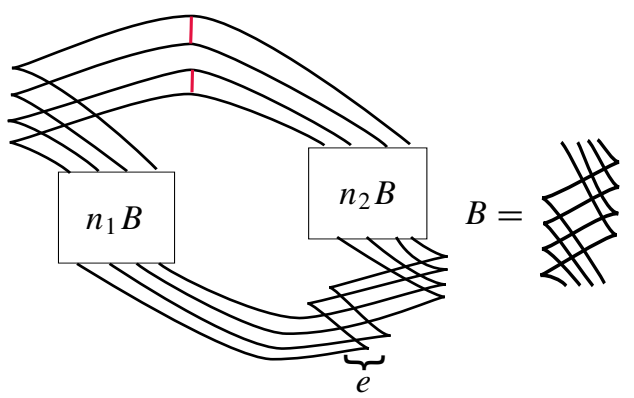

Figure 12. The general form of a maximal tb representative of a negative torus knot $T(-p, 2 k)$, with $p>2 k>0$, with $k=2$ and $|p|=\left(1+n_{1}+n_{2}\right) 2 k+e$; the indicated $k$ surgeries produce a Legendrian trivial link of maximal tb unknots. 


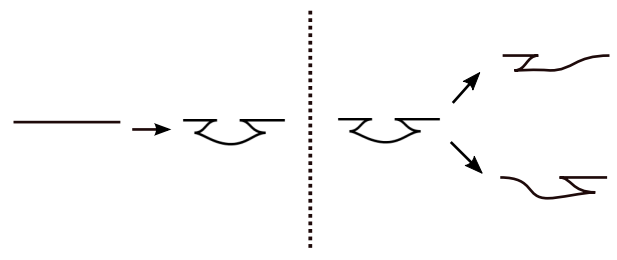

Figure 13. It is possible to construct exact, nonorientable Lagrangian cobordisms between $\Lambda_{+}=\Lambda$ and $S_{-} S_{+}(\Lambda)$, left, and between $\Lambda_{+}=S_{-} S_{+}(\Lambda)$ and $\Lambda_{-}=S_{+}(\Lambda)$ or $\Lambda_{-}=S_{-}(\Lambda)$, right.

\section{Constructions of exact, nonorientable Lagrangian cobordisms}

We will construct an exact, nonorientable Lagrangian endocobordism of crosscap genus 4 for any stabilized Legendrian knot, and a nonorientable Lagrangian cobordism between any two stabilized Legendrian knots. All these exact Lagrangian cobordisms are constructed through isotopy and surgery; see the remark on page 326.

Central to these constructions will be the following lemma, which says that one can always introduce a pair of "oppositely oriented" zig-zags to $\Lambda_{+}$, and if one has a pair of oppositely oriented zig-zags in $\Lambda_{+}$, then one can remove either element of this pair; see Figure 13. One needs to be careful when discussing orientations for the ends of a nonorientable Lagrangian cobordism: given an orientation on $\Lambda_{+}$, there is no canonical orientation for $\Lambda_{-}$. However, although an orientation is needed on $\Lambda$ to distinguish between $S_{+}(\Lambda)$ and $S_{-}(\Lambda), S_{-} S_{+}(\Lambda)$ is a well-defined operation on unoriented knots.

Lemma 5.1. Let $\Lambda$ be a Legendrian knot. Then there exists an exact, nonorientable Lagrangian cobordism

(1) of cross-cap genus 2 between $\Lambda_{+}=\Lambda$ and $\Lambda_{-}=S_{-} S_{+}(\Lambda)$,

(2) of cross-cap genus 1 between $\Lambda_{+}=S_{-} S_{+}(\Lambda)$ and $\Lambda_{-}=S_{+}(\Lambda)$ or $\Lambda_{-}=S_{-}(\Lambda)$.

Proof. The strategy will be to construct the desired exact, nonorientable Lagrangian cobordism via Legendrian isotopy and surgeries that are performed locally, near the site of the stabilizations. Figure 14 illustrates the isotopy and surgeries, the second of which is nonorientable, that imply the existence of a cross-cap genus 2 Lagrangian cobordism between $\Lambda_{+}=\Lambda$ and $\Lambda_{-}=S_{-} S_{+}(\Lambda)$. Figure 15 illustrates the isotopy and surgery that imply the existence of a cross-cap genus 1 Lagrangian cobordism between $\Lambda_{+}=S_{-} S_{+}(\Lambda)$ and $\Lambda_{-}=S_{+}(\Lambda)$, when the original strand is oriented from right to left, or $\Lambda_{-}=S_{-}(\Lambda)$, when the original strand is oriented from left to right.

Exact, nonorientable Lagrangian endocobordisms. In Theorem 1.2, it was shown that Legendrians that are exactly fillable do not have exact, nonorientable Lagrangian 


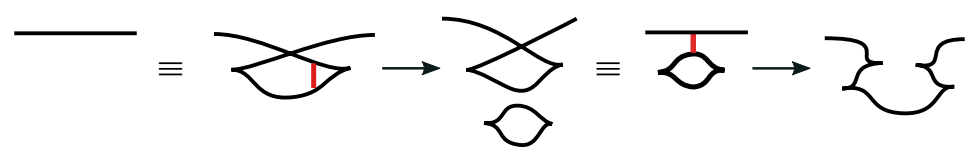

Figure 14. By applying an orientable and a nonorientable surgery, any strand can have a pair of oppositely oriented zig-zags introduced.

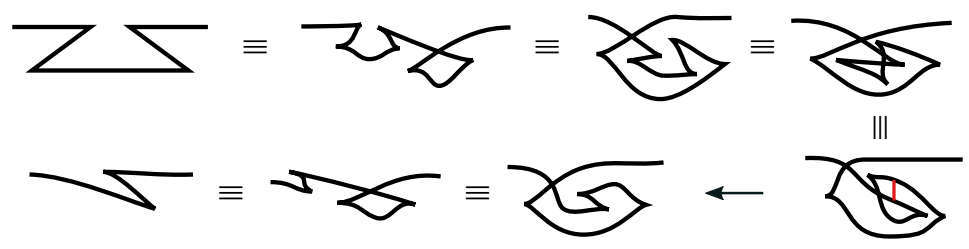

Figure 15. In the presence of oppositely oriented zig-zags, via one nonorientable surgery, one of the zig-zags can be removed.

endocobordisms. However exact, nonorientable Lagrangian endocobordisms do exist for stabilized knots:

Proof of Theorem 1.4. First consider the case where $\Lambda$ is the negative stabilization of a Legendrian: $\Lambda=S_{-}(\hat{\Lambda})$. Then by Lemma 5.1 , there exists an exact, nonorientable Lagrangian cobordism

(1) of cross-cap genus 2 between $\Lambda$ and $S_{-} S_{+}(\Lambda)$,

(2) of cross-cap genus 1 between $S_{-} S_{+}(\Lambda)$ and $S_{+}(\Lambda)$,

(3) of cross-cap genus 1 between $S_{+}(\Lambda)=S_{+}\left(S_{-}(\hat{\Lambda})\right)$ and $S_{-}(\hat{\Lambda})=\Lambda$.

Stacking these cobordisms results in an exact, nonorientable Lagrangian endocobordism of cross-cap genus 4 . Additional stacking results in arbitrary multiples of cross-cap genus 4 .

An analogous argument proves the case where $\Lambda$ is the positive stabilization of a Legendrian: $\Lambda=S_{+}(\hat{\Lambda})$.

\section{Exact, nonorientable Lagrangian cobordisms between stabilized Legendrians.}

Given that every stabilized Legendrian knot has a nonorientable Lagrangian endocobordism, a natural question is: What Legendrian knots can appear as a "slice" of such an endocobordism? We show that any stabilized Legendrian knot can appear as such a slice.

Theorem 5.2. For smooth knot types $K, K^{\prime}$, let $\Lambda$ be any Legendrian representative of $K$ and let $\Lambda^{\prime}$ be a stabilized Legendrian representative of $K^{\prime}$. Then there exists an exact, nonorientable Lagrangian cobordism between $\Lambda_{+}=\Lambda$ and $\Lambda_{-}=\Lambda^{\prime}$. 
Before moving to the proof of Theorem 5.2, we show that nonorientable Lagrangian cobordisms define an equivalence relation on the set of stabilized Legendrian knots:

Proof of Theorem 1.6. Let $\mathcal{L}^{s}$ denote the set of all stabilized Legendrian knots of any smooth knot type. Define the relation $\sim$ on $\mathcal{L}^{s}$ by $\Lambda_{1} \sim \Lambda_{2}$ if there exists an exact, nonorientable Lagrangian cobordism from $\Lambda_{+}=\Lambda_{1}$ to $\Lambda_{-}=\Lambda_{2}$. Reflexivity of $\sim$ follows from Theorem 1.4, symmetry follows from Theorem 5.2, and transitivity follows from Lemma 2.2. Thus $\sim$ defines an equivalence relation. Moreover, by Theorem 5.2, we see that with respect to this equivalence relation, there is only one equivalence class.

To prove Theorem 5.2, it will be useful to first show that there is an exact, nonorientable Lagrangian cobordism between any two stabilized Legendrians of a fixed knot type:

Proposition 5.3. Let $K$ be any smooth knot type, and let $\Lambda, \Lambda^{\prime}$ be Legendrian representatives of $K$, where $\Lambda^{\prime}$ is stabilized. Then there exists an exact, nonorientable Lagrangian cobordism between $\Lambda_{+}=\Lambda$ and $\Lambda_{-}=\Lambda^{\prime}$.

Proof. Fix a smooth knot type $K$, and let $\Lambda_{1}, \Lambda_{2}$ be Legendrian representatives, where $\Lambda_{2}$ is stabilized. By results of Fuchs and Tabachnikov [1997], we know that there exist $r_{1}, \ell_{1}, r_{2}, \ell_{2}$ such that

$$
S_{-}^{\ell_{1}} S_{+}^{r_{1}}\left(\Lambda_{1}\right)=S_{-}^{\ell_{2}} S_{+}^{r_{2}}\left(\Lambda_{2}\right) .
$$

By applying additional positive stabilizations, if needed, we can assume $r_{1}>\ell_{1}$.

Consider the case where $\Lambda_{2}$ is the negative stabilization of some Legendrian: $\Lambda_{2}=S_{-}\left(\hat{\Lambda}_{2}\right)$. By applications of Lemma 5.1, there exists an exact, nonorientable Lagrangian cobordism between

(1) $\Lambda_{1}$ and $S_{-}^{r_{1}} S_{+}^{r_{1}}\left(\Lambda_{1}\right)$,

(2) $S_{-}^{r_{1}} S_{+}^{r_{1}}\left(\Lambda_{1}\right)$ and $S_{-}^{\ell_{1}} S_{+}^{r_{1}}\left(\Lambda_{1}\right)$, and thus between $S_{-}^{r_{1}} S_{+}^{r_{1}}\left(\Lambda_{1}\right)$ and $S_{-}^{\ell_{2}} S_{+}^{r_{2}}\left(\Lambda_{2}\right)$,

(3) $S_{-}^{\ell_{2}} S_{+}^{r_{2}}\left(\Lambda_{2}\right)$ and $S_{+}^{r_{2}}\left(\Lambda_{2}\right)$,

(4) $S_{+}^{r_{2}}\left(\Lambda_{2}\right)=S_{+}^{r_{2}}\left(S_{-}\left(\hat{\Lambda}_{2}\right)\right)$ and $S_{-}\left(\hat{\Lambda}_{2}\right)=\Lambda_{2}$.

By stacking these cobordisms (Lemma 2.2), we have our desired exact, nonorientable Lagrangian cobordism between $\Lambda_{1}$ and $\Lambda_{2}$. An analogous argument proves the case where $\Lambda_{2}$ is the positive stabilization of some Legendrian.

Proof of Theorem 5.2. The strategy here is to first show that one can construct an exact, nonorientable Lagrangian cobordism between $\Lambda$ and a stabilized Legendrian unknot $\Lambda_{0}$. Similarly, it is possible to construct an exact, nonorientable Lagrangian cobordism between $\Lambda^{\prime}$ and a stabilized Legendrian unknot $\Lambda_{0}^{\prime}$; we will show it 


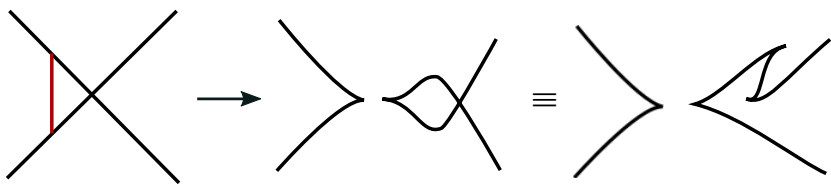

Figure 16. For any Legendrian knot $\Lambda$, perform a surgery near each crossing to get a set of disjoint Legendrian unknots.

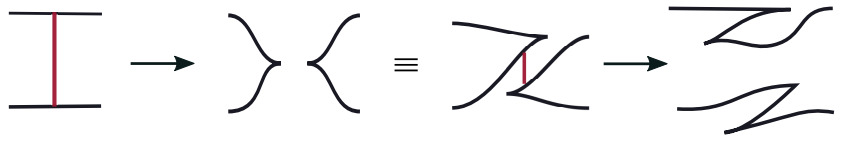

Figure 17. Surgeries used to convert to a link of Legendrian unknots can be "undone", at the cost of additional stabilizations.

is possible to "reverse" this sequence of surgeries and construct an exact, nonorientable Lagrangian cobordism between $\Lambda_{0}^{\prime}$ and $\widetilde{\Lambda}^{\prime}$, which is a stabilization of $\Lambda^{\prime}$. By Proposition 5.3, there exists an exact, nonorientable Lagrangian cobordism between $\Lambda_{0}$ and $\Lambda_{0}^{\prime}$ and between $\tilde{\Lambda}^{\prime}$ and $\Lambda^{\prime}$. Thus we will have the desired exact, nonorientable Lagrangian cobordism between $\Lambda$ and $\Lambda^{\prime}$ by stacking the cobordisms between $\Lambda$ and $\Lambda_{0}$, between $\Lambda_{0}$ and $\Lambda_{0}^{\prime}$, between $\Lambda_{0}^{\prime}$ and $\widetilde{\Lambda}^{\prime}$, and between $\widetilde{\Lambda}^{\prime}$ and $\Lambda^{\prime}$.

We first show how it is possible to construct an exact, nonorientable Lagrangian cobordism from $\Lambda$ to a Legendrian unknot; cf. [Boranda et al. 2013]. Let $\Lambda$ be an arbitrary Legendrian knot. We can assume that $\Lambda$ has at least one positive crossing by, if necessary, applying a Legendrian Reidemeister 1 move. As shown in Figure 16, performing an orientable or nonorientable surgery near a crossing produces a crossing that can be removed through Legendrian Reidemeister moves. Perform such a surgery on every crossing in $\Lambda$ until you have obtained $k$ disjoint stabilized Legendrian unknots; since $\Lambda$ has at least one positive crossing, we have performed at least one nonorientable surgery. Align the $k$ Legendrian unknots vertically and perform surgeries so that we obtain a single stabilized Legendrian unknot $\Lambda_{0}$. In this way, we have constructed an exact, nonorientable Lagrangian cobordism between $\Lambda$ and $\Lambda_{0}$.

A similar procedure can be used to construct a sequence of surgeries from $\Lambda^{\prime}$ to another Legendrian unknot $\Lambda_{0}^{\prime}$; now we show it is possible to "reverse" this procedure and construct a sequence of surgeries from $\Lambda_{0}^{\prime}$ to $\widetilde{\Lambda}^{\prime}$, a Legendrian obtained by applying stabilizations to $\Lambda^{\prime}$. Figure 17 illustrates how every surgery that was used to get to a Legendrian unknot can be undone at the cost of adding additional zig-zags into the original strands. Figure 18 illustrates this procedure with an example. 

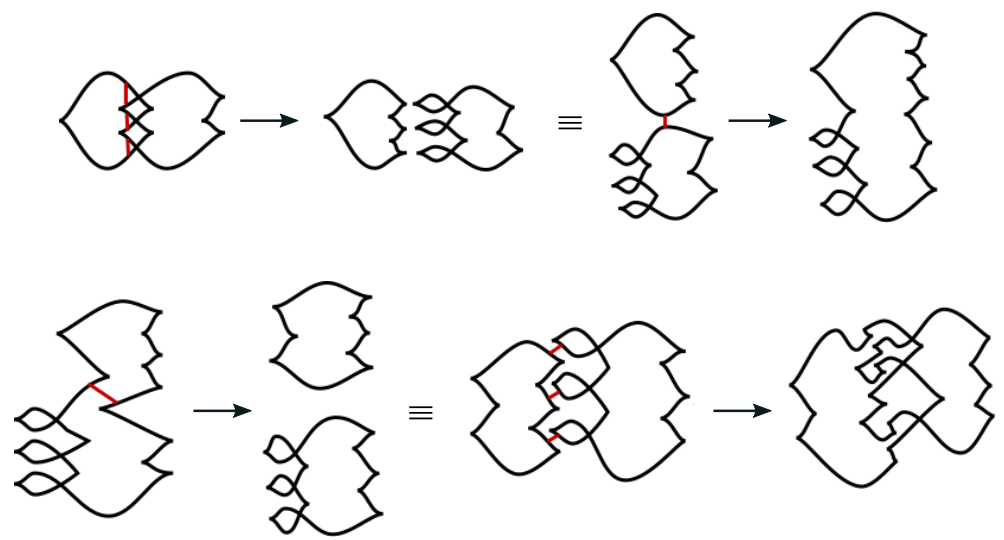

Figure 18. Top: surgeries that give rise to an exact, nonorientable Lagrangian cobordism from the max tb version of $3_{1}$ to a stabilized unknot. Bottom: surgeries that give rise to an exact, nonorientable Lagrangian cobordism from the stabilized unknot to a stabilized representative of $3_{1}$.

As outlined at the beginning of the proof, these constructions prove the existence of an exact Lagrangian cobordism from $\Lambda_{+}=\Lambda$ to $\Lambda_{-}=\Lambda^{\prime}$.

\section{Additional questions}

We end with a brief discussion of some additional questions.

From results above, we know that exactly fillable Legendrian knots do not admit exact, nonorientable Lagrangian endocobordisms, while stabilized Legendrian knots do. There are examples of Legendrian knots that are neither exactly fillable nor stabilized. As mentioned above, Ekholm [2008] has shown that if $\Lambda$ is exactly fillable, then there exists an ungraded augmentation of $\mathcal{A}(\Lambda)$. By work of Sabloff [2005] and, independently, Fuchs and Ishkhanov [2004], we then know that there exists an ungraded ruling of $\Lambda$. (Definitions of graded and ungraded rulings can be found, for example, in [Kálmán 2008].) Then it follows by [Rutherford 2006] that the Kauffman bound on the maximal tb value for all Legendrian representatives of the smooth knot type of $\Lambda$ is sharp. Thus, if the Kauffman bound is not sharp for the smooth knot type $K$, then no Legendrian representative of $K$ is exactly fillable. So a natural question is:

Question 6.1. If $\Lambda$ is a maximal tb representative of a knot type $K$ for which the upper bound on tb for all Legendrian representatives given by the Kauffman polynomial is not sharp, does $\Lambda$ have an exact, nonorientable Lagrangian endocobordism? 
The Legendrian representative of $m\left(8_{19}\right)$ shown in Figure 1 satisfies the hypotheses in Question 6.1; the Kauffman bound is known to be sharp for all knots with 10 or fewer crossings except $m\left(8_{19}\right), m\left(9_{42}\right), m\left(10_{124}\right), m\left(10_{128}\right), m\left(10_{132}\right)$, and $m\left(10_{136}\right)$ [ $\mathrm{Ng} 2001 ; 2005$; Rutherford 2006]. As mentioned in the Introduction, [Chantraine et al. 2015] contains results which imply that the answer to Question 6.1 is no. However, this now spawns new questions. For example, consider the max tb Legendrian representative of $m\left(10_{132}\right)$ given as $K_{2}$ in [Sivek 2013, Figure 2]. The Legendrian $K_{2}$ is not stabilized, does not have an augmentation (and thus is not exactly fillable), and does not have a finite-dimensional representation. Does $K_{2}$ have an exact, nonorientable Lagrangian endocobordism?

There are also examples of Legendrians with nonmaximal tb that are not stabilized. For example, $m\left(10_{161}\right)$ is a knot type where the unique maximal tb representative has a filling. However, there is a Legendrian representative with nonmaximal tb that does not arise as a stabilization. As shown in [Shonkwiler and Vela-Vick 2011, Figure 1], this nonmaximal tb, nonstabilized Legendrian does have an ungraded ruling, and the characteristic algebra of $K_{2}$ does not have a finite-dimensional representation [Sivek 2013].

Question 6.2. Does the nonstabilized, nonmaximal tb Legendrian representative of $m\left(10_{161}\right)$ have an exact, nonorientable Lagrangian endocobordism?

Additional examples of nonstabilized and nonmaximal tb representatives can be found in the Legendrian knot atlas of Chongchitmate and $\mathrm{Ng}$ [2013].

There are additional questions that arise from the constructions of fillings. For example, it is known by results of Chantraine [2010] that orientable fillings realize the smooth 4-ball genus. In Figure 6, examples are given of nonorientable Lagrangian fillings of maximal tb representatives of $\sigma_{2}$ and $m\left(\sigma_{2}\right)$ of cross-cap genus 2 and 4, respectively: the smooth 4-dimensional cross-cap number of both $6_{2}$ and $m\left(6_{2}\right)$ is 1 .

Question 6.3. Does there exist a nonorientable Lagrangian filling of these Legendrian representatives of $6_{2}$ and $m\left(6_{2}\right)$ of cross-cap genus 1 ?

\section{Acknowledgements}

We thank Baptiste Chantraine, Richard Hind, and Josh Sabloff for stimulating discussions. We also thank Georgios Dimitroglou Rizell and Tobias Ekholm for helpful comments. We are also grateful to the referee for numerous comments and suggestions that made the exposition better. Both authors thank the Andrew W. Mellon Foundation for supporting the first author with a Mellon Mays Undergraduate Fellowship; this paper grew out of her thesis project [Capovilla-Searle 2015]. Traynor gratefully acknowledges the hospitality of the Institute for Advanced Study 
in Princeton and support at IAS from The Fund for Mathematics during a portion of this work.

\section{References}

[Audin 1988] M. Audin, "Fibrés normaux d'immersions en dimension double, points doubles d'immersions lagragiennes et plongements totalement réels", Comment. Math. Helv. 63:4 (1988), 593-623. MR 966952 Zbl 0666.57024

[Audin 1990] M. Audin, "Quelques remarques sur les surfaces lagrangiennes de Givental”, J. Geom. Phys. 7:4 (1990), 583-598. MR 1131914 Zbl 0728.57020

[Audin et al. 1994] M. Audin, F. Lalonde, and L. Polterovich, "Symplectic rigidity: Lagrangian submanifolds", pp. 271-321 in Holomorphic curves in symplectic geometry, edited by M. Audin and J. Lafontaine, Progr. Math. 117, Birkhäuser, Basel, 1994. MR 1274934 Zbl 0802.53001

[Baldwin and Sivek 2014] J. A. Baldwin and S. Sivek, "Invariants of Legendrian and transverse knots in monopole knot homology", preprint, 2014. arXiv 1405.3275

[Boranda et al. 2013] B. Boranda, L. Traynor, and S. Yan, "The surgery unknotting number of Legendrian links", Involve 6:3 (2013), 273-299. MR 3101761 Zbl 06227497

[Bourgeois et al. 2015] F. Bourgeois, J. M. Sabloff, and L. Traynor, "Lagrangian cobordisms via generating families: construction and geography", Algebr. Geom. Topol. 15:4 (2015), 2439-2477. MR 3402346 Zbl 1330.57037

[Capovilla-Searle 2015] O. Capovilla-Searle, Surgery strings and loops of Legendrian knots, bachelor's thesis, Bryn Mawr College, 2015.

[Chantraine 2010] B. Chantraine, "Lagrangian concordance of Legendrian knots", Algebr. Geom. Topol. 10:1 (2010), 63-85. MR 2580429 Zbl 1203.57010

[Chantraine 2015] B. Chantraine, "Lagrangian concordance is not a symmetric relation", Quantum Topol. 6:3 (2015), 451-474. MR 3392961 Zbl 1337.57056

[Chantraine et al. 2015] B. Chantraine, G. Dimitroglou Rizell, P. Ghiggini, and R. Golovko, "Floer theory for Lagrangian cobordisms", preprint, 2015. arXiv 1511.09471v1

[Chekanov 2002] Y. Chekanov, "Differential algebra of Legendrian links", Invent. Math. 150:3 (2002), 441-483. MR 1946550 Zbl 1029.57011

[Chongchitmate and Ng 2013] W. Chongchitmate and L. Ng, "An atlas of Legendrian knots", Exp. Math. 22:1 (2013), 26-37. MR 3038780 Zbl 1267.57004

[Cornwell et al. 2016] C. Cornwell, L. Ng, and S. Sivek, "Obstructions to Lagrangian concordance", Algebr. Geom. Topol. 16:2 (2016), 797-824. MR 3493408 Zbl 06577067

[Dimitroglou Rizell 2014] G. Dimitroglou Rizell, "Legendrian ambient surgery and Legendrian contact homology", preprint, 2014. arXiv 1205.5544v5

[Dimitroglou Rizell 2016] G. Dimitroglou Rizell, "Lifting pseudo-holomorphic polygons to the symplectisation of $P \times \mathbb{R}$ and applications", Quantum Topol. 7:1 (2016), 29-105. MR 3459958 Zbl 06558091

[Ekholm 2008] T. Ekholm, "Rational symplectic field theory over $\mathbb{Z}_{2}$ for exact Lagrangian cobordisms”, J. Eur. Math. Soc. (JEMS) 10:3 (2008), 641-704. MR 2421157 Zbl 1154.57020

[Ekholm 2012] T. Ekholm, "Rational SFT, linearized Legendrian contact homology, and Lagrangian Floer cohomology", pp. 109-145 in Perspectives in analysis, geometry, and topology, edited by I. Itenberg et al., Progr. Math. 296, Birkhäuser/Springer, New York, 2012. MR 2884034 Zbl 1254.57024 
[Ekholm et al. 2007] T. Ekholm, J. Etnyre, and M. Sullivan, "Legendrian contact homology in $P \times \mathbb{R}$ ", Trans. Amer. Math. Soc. 359:7 (2007), 3301-3335. MR 2299457 Zbl 1119.53051

[Ekholm et al. 2012] T. Ekholm, K. Honda, and T. Kálmán, "Legendrian knots and exact Lagrangian cobordisms", preprint, 2012. arXiv 1212.1519

[Eliashberg 1998] Y. Eliashberg, "Invariants in contact topology", Doc. Math. Extra Vol. II (1998), 327-338. MR 1648083 Zbl 0913.53010

[Eliashberg and Gromov 1998] Y. Eliashberg and M. Gromov, "Lagrangian intersection theory: finitedimensional approach", pp. 27-118 in Geometry of differential equations, edited by A. Khovanskiu et al., Amer. Math. Soc. Transl. Ser. 2 186, Amer. Math. Soc., Providence, RI, 1998. MR 1732407 Zbl 0919.58015

[Eliashberg et al. 2000] Y. Eliashberg, A. Givental, and H. Hofer, "Introduction to symplectic field theory", Geom. Funct. Anal. Special Vol., Part II (2000), 560-673. MR 1826267 Zbl 0989.81114

[Etnyre 2003] J. B. Etnyre, "Introductory lectures on contact geometry", pp. 81-107 in Topology and geometry of manifolds (Athens, GA, 2001), edited by G. Matić and C. McCrory, Proc. Sympos. Pure Math. 71, Amer. Math. Soc., Providence, RI, 2003. MR 2024631 Zbl 1045.57012

[Etnyre 2005] J. B. Etnyre, "Legendrian and transversal knots", pp. 105-185 in Handbook of knot theory, edited by W. Menasco and M. Thistlethwaite, Elsevier, Amsterdam, 2005. MR 2179261 Zbl 1095.57006

[Etnyre and Honda 2001] J. B. Etnyre and K. Honda, "Knots and contact geometry, I: Torus knots and the figure eight knot”, J. Symplectic Geom. 1:1 (2001), 63-120. MR 1959579 Zbl 1037.57021

[Etnyre et al. 2013] J. B. Etnyre, L. L. Ng, and V. Vértesi, "Legendrian and transverse twist knots", J. Eur. Math. Soc. (JEMS) 15:3 (2013), 969-995. MR 3085098 Zbl 1271.57060

[Fuchs and Ishkhanov 2004] D. Fuchs and T. Ishkhanov, "Invariants of Legendrian knots and decompositions of front diagrams", Mosc. Math. J. 4:3 (2004), 707-717, 783. MR 2119145 Zbl 1073.53106

[Fuchs and Tabachnikov 1997] D. Fuchs and S. Tabachnikov, "Invariants of Legendrian and transverse knots in the standard contact space", Topology 36:5 (1997), 1025-1053. MR 1445553 Zbl 0904.57006

[Givental 1986] A. B. Givental', "Lagrangian imbeddings of surfaces and the open Whitney umbrella", Funktsional. Anal. i Prilozhen. 20:3 (1986), 35-41, 96. In Russian; translated in Funct. Anal. Appl. 20:3 (1986), 197-203. MR 868559 Zbl 0621.58025

[Gromov 1985] M. Gromov, "Pseudoholomorphic curves in symplectic manifolds", Invent. Math. 82:2 (1985), 307-347. MR 809718 Zbl 0592.53025

[Hayden and Sabloff 2015] K. Hayden and J. M. Sabloff, "Positive knots and Lagrangian fillability", Proc. Amer. Math. Soc. 143:4 (2015), 1813-1821. MR 3314092 Zbl 1311.57033

[Kálmán 2008] T. Kálmán, "Rulings of Legendrian knots as spanning surfaces", Pacific J. Math. 237:2 (2008), 287-297. MR 2421123 Zbl 1153.53058

[Lalonde and Sikorav 1991] F. Lalonde and J.-C. Sikorav, "Sous-variétés lagrangiennes et lagrangiennes exactes des fibrés cotangents", Comment. Math. Helv. 66:1 (1991), 18-33. MR 1090163 Zbl 0759.53022

[McDuff and Salamon 1998] D. McDuff and D. Salamon, Introduction to symplectic topology, 2nd ed., Clarendon/Oxford University Press, New York, 1998. MR 1698616 Zbl 1066.53137

[Nemirovskiı̌ 2009] S. Y. Nemirovskiŭ, "The homology class of a Lagrangian Klein bottle", Izv. Ross. Akad. Nauk Ser. Mat. 73:4 (2009), 37-48. In Russian; translated in Izv. Math. 73:4 (2009), 689-698. MR 2583965 Zbl 1191.57019 
[Ng 2001] L. L. Ng, "Maximal Thurston-Bennequin number of two-bridge links", Algebr. Geom. Topol. 1 (2001), 427-434. MR 1852765 Zbl 1056.57010

[Ng 2005] L. Ng, "A Legendrian Thurston-Bennequin bound from Khovanov homology", Algebr. Geom. Topol. 5 (2005), 1637-1653. MR 2186113 Zbl 1091.57009

[Polterovich 1991] L. Polterovich, "The surgery of Lagrange submanifolds", Geom. Funct. Anal. 1:2 (1991), 198-210. MR 1097259 Zbl 0754.57027

[Rutherford 2006] D. Rutherford, "Thurston-Bennequin number, Kauffman polynomial, and ruling invariants of a Legendrian link: the Fuchs conjecture and beyond", Int. Math. Res. Not. 2006 (2006), Art. ID 78591. MR 2219227 Zbl 1106.57012

[Sabloff 2003] J. M. Sabloff, "Invariants of Legendrian knots in circle bundles", Commun. Contemp. Math. 5:4 (2003), 569-627. MR 2003211 Zbl 1046.57013

[Sabloff 2005] J. M. Sabloff, "Augmentations and rulings of Legendrian knots", Int. Math. Res. Not. 2005:19 (2005), 1157-1180. MR 2147057 Zbl 1082.57020

[Sabloff and Traynor 2013] J. M. Sabloff and L. Traynor, "Obstructions to Lagrangian cobordisms between Legendrians via generating families", Algebr. Geom. Topol. 13:5 (2013), 2733-2797. MR 3116302 Zbl 1270.53096

[Shevchishin 2009] V. V. Shevchishin, "Lagrangian embeddings of the Klein bottle and the combinatorial properties of mapping class groups", Izv. Ross. Akad. Nauk Ser. Mat. 73:4 (2009), 153-224. In Russian; translated in Izv. Math. 73:4 (2009), 797-859. MR 2583968 Zbl 1196.57021

[Shonkwiler and Vela-Vick 2011] C. Shonkwiler and D. S. Vela-Vick, "Legendrian contact homology and nondestabilizability”, J. Symplectic Geom. 9:1 (2011), 33-44. MR 2787360 Zbl 1226.57013

[Sivek 2013] S. Sivek, "The contact homology of Legendrian knots with maximal Thurston-Bennequin invariant”, J. Symplectic Geom. 11:2 (2013), 167-178. MR 3046489 Zbl 1277.53082

Received September 27, 2015. Revised April 19, 2016.

Orsola CAPOVILla-SEARLE

DEPARTMENT OF MATHEMATICS

DUKE UNIVERSITY

DURHAM, NC 27708

UNITED STATES

orsola.capovilla.searle@duke.edu

LISA TRAYNOR

DEPARTMENT OF MATHEMATICS

BRYN MAWR COLLEGE

BRYN MAWR, PA 19010

UNITED STATES

ltraynor@brynmawr.edu 


\title{
PACIFIC JOURNAL OF MATHEMATICS
}

Founded in 1951 by E. F. Beckenbach (1906-1982) and F. Wolf (1904-1989)

$$
\text { msp.org/pjm }
$$

\section{EDITORS}

\author{
Don Blasius (Managing Editor) \\ Department of Mathematics \\ University of California \\ Los Angeles, CA 90095-1555 \\ blasius@math.ucla.edu
}

\author{
Paul Balmer \\ Department of Mathematics \\ University of California \\ Los Angeles, CA 90095-1555 \\ balmer@math.ucla.edu \\ Robert Finn \\ Department of Mathematics \\ Stanford University \\ Stanford, CA 94305-2125 \\ finn@math.stanford.edu \\ Sorin Popa \\ Department of Mathematics \\ University of California \\ Los Angeles, CA 90095-1555 \\ popa@math.ucla.edu
}

\author{
Vyjayanthi Chari \\ Department of Mathematics \\ University of California \\ Riverside, CA 92521-0135 \\ chari@math.ucr.edu \\ Kefeng Liu \\ Department of Mathematics \\ University of California \\ Los Angeles, CA 90095-1555 \\ liu@math.ucla.edu \\ Igor Pak \\ Department of Mathematics \\ University of California \\ Los Angeles, CA 90095-1555 \\ pak.pjm@gmail.com \\ Paul Yang \\ Department of Mathematics \\ Princeton University \\ Princeton NJ 08544-1000 \\ yang@math.princeton.edu
}

\section{PRODUCTION}

Silvio Levy, Scientific Editor, production@msp.org

\section{SUPPORTING INSTITUTIONS}

ACADEMIA SINICA, TAIPEI

CALIFORNIA INST. OF TECHNOLOGY

STANFORD UNIVERSITY

UNIV. OF BRITISH COLUMBIA

UNIV. OF CALIFORNIA, BERKELEY

UNIV. OF CALIFORNIA, DAVIS

UNIV. OF CALIFORNIA, LOS ANGELES

UNIV. OF CALIFORNIA, RIVERSIDE

UNIV. OF CALIFORNIA, SAN DIEGO

UNIV. OF CALIF., SANTA BARBARA
KEIO UNIVERSITY

MATH. SCIENCES RESEARCH INSTITUTE

NEW MEXICO STATE UNIV.

OREGON STATE UNIV.
Daryl Cooper

Department of Mathematics

University of California

Santa Barbara, CA 93106-3080 cooper@math.ucsb.edu

Jiang-Hua Lu

Department of Mathematics

The University of Hong Kong

Pokfulam Rd., Hong Kong

jhlu@maths.hku.hk

$$
\text { Jie Qing }
$$

Department of Mathematics

University of California

Santa Cruz, CA 95064

qing@ cats.ucsc.edu

\author{
UNIV. OF CALIF., SANTA CRUZ \\ UNIV. OF MONTANA \\ UNIV. OF OREGON \\ UNIV. OF SOUTHERN CALIFORNIA \\ UNIV. OF UTAH \\ UNIV. OF WASHINGTON \\ WASHINGTON STATE UNIVERSITY
}

These supporting institutions contribute to the cost of publication of this Journal, but they are not owners or publishers and have no responsibility for its contents or policies.

See inside back cover or msp.org/pjm for submission instructions.

The subscription price for 2016 is US $\$ 440 /$ year for the electronic version, and \$600/year for print and electronic.

Subscriptions, requests for back issues and changes of subscriber address should be sent to Pacific Journal of Mathematics, P.O. Box 4163, Berkeley, CA 94704-0163, U.S.A. The Pacific Journal of Mathematics is indexed by Mathematical Reviews, Zentralblatt MATH, PASCAL CNRS Index, Referativnyi Zhurnal, Current Mathematical Publications and Web of Knowledge (Science Citation Index).

The Pacific Journal of Mathematics (ISSN 0030-8730) at the University of California, c/o Department of Mathematics, 798 Evans Hall \#3840, Berkeley, CA 94720-3840, is published twelve times a year. Periodical rate postage paid at Berkeley, CA 94704, and additional mailing offices. POSTMASTER: send address changes to Pacific Journal of Mathematics, P.O. Box 4163, Berkeley, CA 94704-0163.

PJM peer review and production are managed by EditFLOW ${ }^{\circledR}$ from Mathematical Sciences Publishers.

PUBLISHED BY

\section{I. mathematical sciences publishers}

nonprofit scientific publishing

http://msp.org/

(C) 2016 Mathematical Sciences Publishers 


\section{PACIFIC JOURNAL OF MATHEMATICS}

Volume $285 \quad$ No. $2 \quad$ December 2016

The $\mathrm{SU}(N)$ Casson-Lin invariants for links

HANS U. BODEN and ERIC HARPER

The SU(2) Casson-Lin invariant of the Hopf link

HANS U. BODEN and CHRISTOPHER M. HERALD

Commensurations and metric properties of Houghton's groups

José BuRILlo, SEAn Cleary, ARMANDo MaRTino and ClaAs

E. RÖVER

Conformal holonomy equals ambient holonomy

ANDREAS ČAP, A. RoD GOVER, C. RoBIN GRAHAM and

MATTHIAS HAMMERL

Nonorientable Lagrangian cobordisms between Legendrian knots

ORSOLA CAPOVILLA-SEARLE and LISA TRAYNOR

A strong multiplicity one theorem for $\mathrm{SL}_{2}$

JINGSONG CHAI and QING ZHANG

The Yamabe problem on noncompact CR manifolds

PAK TUNG Ho and SeONGTAG KIM

Isometry types of frame bundles

WOUTER VAN LIMBEEK

Bundles of spectra and algebraic K-theory

JOHN A. LIND

Hidden symmetries and commensurability of 2-bridge link complements

CHRISTIAN MiLlichaP and WILLIAM WORDEN

On seaweed subalgebras and meander graphs in type $C$

DMITRI I. PANYUSHEV and OKSANA S. YAKIMOVA

The genus filtration in the smooth concordance group 